\section{Righting the wrong protein}

\author{
Paul M. Quinton
}

IN the summer of 1989 the gene for the fatal hereditary disease, cystic fibrosis (CF), was identified and sequenced ${ }^{1-3}$. Now, barely a year later, two independent groups simultaneously report correction of CF cells transfected with the normal gene. One group (Drumm et al.) reports its gene complementation results in $\mathrm{Cell}^{4}$. The other presents its work on expressing and synthesizing the gene product ${ }^{5}$ and its complementation work ${ }^{6}$ in next week's Nature.

Gregory et al. ${ }^{5}$ describe in vitro and in vivo synthesis of the gene product, cystic fibrosis transmembrane conductance regulator (CFTR). Like Drumm et al., they surmised that previous failures to express the gene were due to toxic overexpression caused by a cryptic bacterial promoter in exon 6B of the CFTR gene. Gregory et al. solved the problem by incorporating the full length of an open reading frame complementary DNA of CFTR into a low-copy-number plasmid, whereas Drumm et al. introduced three mutations into the CFTR gene to neutralize the promoter. Gregory et al. went on to synthesize the CFTR gene product in vitro in cell-free reticulocyte lysates and in vivo with recombinant HeLa cells. To confirm gene expression they developed antibodies to fusion proteins containing peptides encoded by exon 10 and exon 13 of the gene, which labelled protein products of relative molecular mass about 130,000 and $140,000\left(M_{\mathrm{r}} 130 \mathrm{~K}\right.$ and $\left.140 \mathrm{~K}\right)$ from the in vitro and in vivo syntheses, respectively. The different molecular masses are accounted for by in vivo glycosylation. Although these results give convincing evidence of expression of the CFTR protein, the discrepancy between the apparent small size of these products compared to that predicted for the CFTR protein (which consists of 1,480 amino acids and has a putative $M_{\mathrm{r}}$ of $170 \mathrm{~K}$ ) requires clarification.

\section{Transfection}

Rich et al. ${ }^{6}$ used two independent assays to demonstrate that the defect in cyclic AMP-mediated stimulus-response coupling of chloride permeability in a CF respiratory cell line was corrected in most cells when these cells were transfected with a hybrid vaccinia virus-CFTR cDNA plasmid expression system. Failures were attributed to inefficiency in transfection. Few measurements were possible because of tedious technical demands and the short life of cells after viral infection, but the results from both assays taken together offer convincing evidence that the defect was effectively complemented. Drumm et al. corrected the same defect in a pancreatic tumour cell from a $\mathrm{CF}$ patient, using a recombinant retrovirus that produced stable, viable cells and permitted a larger number of measurements using similar assays. Even though these results do not establish how CFTR affects chloride permeability, they do, of course, prove to physiologists what geneticists already knew - that the gene that was identified through 'reverse genetics' is the CF gene.

There are now at least two major routes of research to be pursued directly. First, CFTR antibodies in cells known to express CF defects must be localized cytologically. The many cell types of relatively undesignated function that comprise airway epithelia, and the relative inaccessibility of other equally complicated $\mathrm{CF}$ target tissues, may create confusion with respect to antibody specificity in localization studies, but the simplicity of the sweat gland could provide a standard in such assays. The sweat gland reabsorptive duct is a syncytium of cells expressing the clearest and most consistent defect in CF - impermeability to chloride ions - and the sweat gland secretory coil, which characteristically fails to respond to $\beta$-adrenergic stimulation in $\mathrm{CF}$, is composed of only two very distinct secretory cells. It seems reasonable to insist that any antibody to CFTR should show binding in these two components of the gland. Antibodies meeting this criterion could then be used to identify target cells in the more complicated tissues of the airways, pancreas and other affected organs. Distinct antibodies meeting this criterion (preferably antibodies to different epitopes of CFTR) will be important in determining if other cells not currently recognized to be affected in CF also express CFTR. Antibody-localization studies seem almost certain to provide insights into the allimportant question of the function of the affected protein, which is also at the heart of the second pressing route of pursuit the biochemical properties and function of CFTR.

On the basis of homology data and modelling, Hyde et al. ${ }^{7}$ have argued that CFTR is probably not an ion channel, but rather an ATPase transport protein. The course of further research, and indeed our understanding of the nature of the disease, depends on the assessment of this hypothesis. It predicts that aberrant ionchannel phenomena are secondary consequences of deranged cell metabolism and implies that the disease could be even more amenable to therapy than anticipated if the species transported by CFTR could be identified and controlled. Alternatively, if CFTR is an ion channel or a closely associated channel regulator, correction will probably require supplementing or bypassing with other components.

Now that quantities of CFTR can be produced, several crucial questions can be answered to define its properties and potential function and thereby distinguish between these two main hypotheses. Initially, we must know if the region containing the most common CF defect (a deleted phenylalanine in the 508 position of the CFTR protein) is a nucleotide-binding fold; that is, does it bind ATP or other nucleotides? If so, is it a catalytic site for ATP hydrolysis? And if it is, is it a transport ATPase or a kinase? If it is a kinase, what does it phosphorylate? And certainly, if it is an active transport protein, what does it transport?

\section{Channel regulation}

Even if CFTR does not hydrolyse ATP, nucleotides might still be involved in channel regulation. For example, there is much evidence $^{8}$ showing that the opening of a potassium-ion channel found in pancreatic $\beta$-islet cells, heart and skeletal muscle cells, and some neurons, is highly sensitive to ATP or, more probably, to the ratio of cytoplasmic levels of ATP to ADP (energy charge). Airway epithelia ${ }^{9}$ and sweat ducts ${ }^{10}$ are characterized by chloride conductances (permeabilities) which decrease markedly with energy deprivation. These observations could suggest that the putative nucleotide-binding fold of CFTR determines the sensitivity of an ion channel, directly or indirectly, to cell nucleotide levels, possibly in a way that protects the cell from electrolyte overloads when it is under stress or short of energy.

Whatever the actual function of CFTR, the two groups that have demonstrated expression and complementation of the $\mathrm{CF}$ gene have taken us to another milestone on the route from the genetic defect to effective therapy. Only a few years ago the idea of introducing normal genes into the CF lung to correct its fatal susceptibility to infection was science fiction. Now the accomplishments of these investigators seem to press the fiction inspiringly close to reality.

Paul M. Quinton is in the Division of Biomedical Sciences, University of California, Riverside, California 92521, USA.

1. Rommens, J.M. et al. Science 245, 1059-1065 (1989)

2. Riordan, J.R. et al. Science 245, 1066-1072 (1989).

3. Kerem, B.-S. et al. Science 245, 1073-1080 (1989).

4. Drumm, M.L. et al. Cell 62, 1227 (1990).

5. Gregory, R.J. et al. Nature 347, 382-386 (1990)

6. Rich, D.P. et al. Nature 347, 358-363 (1990).

7. Hyde, S.C. et al. Nature 346, 362-365 (1990)

8. Rorsman, P., Berggren, P.-O., Bokvist, K. \& Efendic, S. News Physiol. Sci. 5, 143-147 (1990).

9. Stutts, M.J., Gatzy, J.T. \& Boucher, R.C. Am. J. Physiol. $64,253-258(1988)$

10. Quinton, P.M. \& Reddy, M.M. in The Identification of the CF (Cystic Fibrosis) Gene: Recent Progress and New Strategies (eds Tsui, L.-C., Romeo, G., Greger, R. \& Gorini, S.) (Plenum, New York, in the press). 